\title{
A Generalization of the Popov Criterion
}

\author{
by J. B. MOORE AND B. D. O. ANDERSON \\ Department of Electrical Engineering \\ University of Newcastle, New South Wales, Australia
}

ABSTRACT: A criterion for the stability of control systems which contain an arbitrary finite number of memoryless nonlinearities is considered. The criterion is such that the degree of stability may be specified, and such that for the case when the absalute stability of a control system having one memoryless nonlinearity is considered, it reduces to the original Popov criterion.

\section{Introduction}

The formulation and proof of absolute stability criteria applicable to systems having many memoryless nonlinearities is given in (1) and (2). The significant contribution of (1) is the demonstration of the existence of a frequency response stability criterion for multiple nonlinearity systems. The contribution of (2) is that means of constructing system Lyapunov functions are given. Also, the convenient application of the network theory concept of a positive real function to the control theory concept of a minimal realization of a transfer function (3) to proving the system stability criteria is indicated. However, consideration of the degree of stability of such systems is not given except in the single nonlinearity case (4).

This paper gives an application of the theorem of (3) to establish a stability criterion for control systems containing an arbitrary finite number of memoryless nonlinearities that is more general than either of those considered in (1) and (2), and states clearly the conditions for which it may be applied. The criterion is such that the degree of stability may be specified.

When the absolute stability of a control system having one memoryless nonlinearity is considered, the criterion reduces to that given originally by Popov (5).

\section{Stability Criterion}

The nonlinear systems considered are those where the system is of the form (or may be arranged to be of the form) of that shown in Fig. 1. Lyapunov stability is considered, hence, the inputs are not indicated. The matrix $W(s)$ is an $n \times n$ matrix of stable rational transfer functions, assumed to be such that

$$
W(\infty)=0 .
$$

The nonlinearities $\mu_{i}\left(y_{i}\right)(i=1,2, \cdots, n)$ are assumed to satisfy the conditions

$$
0 \leq \mu_{i}\left(y_{i}\right) y_{i}<k_{i} y_{i}{ }^{2} \text { for } k_{i}>0(i=1,2, \cdots, n) \text {. }
$$




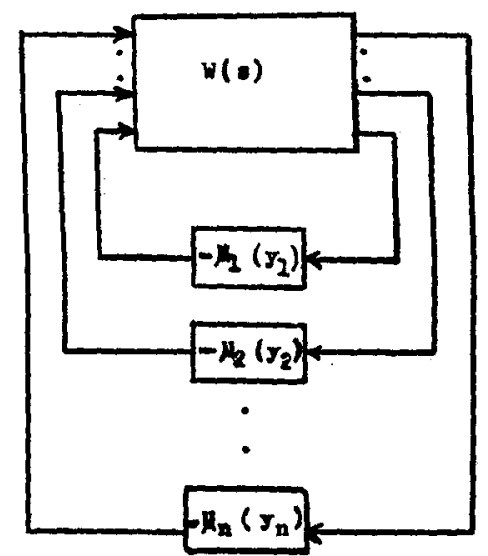

Frg. 1. System with multiple nonlinearities.

The $k_{i}$ define the matrix $K=\operatorname{diag}\left\{k_{1}, k_{2}, \cdots, k_{n}\right\}$, and the $\mu_{i}$ the vector $\mu=$ $\left(\mu_{1}, \mu_{2}, \cdots, \mu_{n}\right)^{\prime}$. The stability criterion for the above system is given as follows:

\section{Theorem.}

Part (a). If there exist real diagonal matrices, $A=\operatorname{diag}\left\{a_{1}, a_{2}, \cdots, a_{n}\right\}$, $B=\operatorname{diag}\left\{b_{1}, b_{2}, \cdots, b_{n}\right\}$, with $a_{i} \geq 0, b_{i} \geq 0, a_{i}+b_{i}>0$, and $-a_{i} / b_{i}$ not a pole of any of the $i$ th row elements of $W(s)$, such that

$$
Z(s)=A K^{-1}+(A+B s) W(s)
$$

is positive real, then the system shown in Fig. 1 is stable.

Part $(b)$. For some real $\sigma_{0}<0$, suppose that the same conditions apply on the elements of $A$ and $B$ as in Part (a), except that $-a_{i} / b_{i}$ must not be a pole of the $i$ th row of $W\left(s+\sigma_{0}\right)$; suppose further that the poles of $W(s)$ lie in the region $\operatorname{Re} s<\sigma_{0}$. If for some $A$ and $B$,

$$
Z_{1}(s)=A K^{-1}+(A+B s) W\left(s+\sigma_{0}\right)
$$

is positive real, then the system is stable. Moreover, if the nonlinearities satisfy

$$
\mu_{i}\left(y_{i}\right) y_{i} \geq \int_{0}^{\nu_{i}} \mu_{i}\left(\rho_{i}\right) d \rho_{i} \quad i=1,2, \cdots, n
$$

there exists a Lyapunov function $V$ for which

$$
\dot{V} / V \leq \sigma_{0}
$$

and if the nonlinearities satisfy

$$
\mu_{i}\left(y_{i}\right) y_{i} \geq 2 \int_{0}^{\nu_{i}} \mu_{i}\left(\rho_{i}\right) d \rho_{i} \quad i=1,2, \cdots, n
$$


there exists a Lyapunov function $V$ for which

$$
\dot{V} / V \leq 2 \sigma_{0}
$$

We comment that condition (2) implies condition (5) for sufficiently small $y_{i}$, and for all monotonic nonlinearities. Condition (2) implies condition (7) for any nonlinearity which is concave upwards. Certainly, for any given nonlinearity the range of $y$ values for which condition (5) or (7) is satisfied can be determined and the theorem applied (in a limited way, admittedly) in this range.

We further comment that the conditions on $A$ and $B$ ensure that the degree of $Z(s)\left[Z_{1}(s)\right](6)$ is the same as that of $W(s)\left[W\left(s+\sigma_{0}\right)\right]$, i.e. that the dimensions of minimal realizations of $Z(s)\left[Z_{1}(s)\right]$ and $W(s)\left[W\left(s+\sigma_{0}\right)\right]$ are the same.

Proof of Theorem. A transfer function $W(s)$ satisfying (1) possesses a minimal realization $\{F, G, H\}$ which is a set of three constant matrices, ( $F$ being $p \times p, G$ and $H$ being $p \times n$ where $p$ is the degree of $W(s))$, satisfying

$$
W(s)=H^{\prime}(s I-F)^{-1} G .
$$

An expansion of $Z_{1}(s)$ in terms of $F, G$ and $H$ gives

$$
\begin{aligned}
Z_{1}(s)= & A K^{-1}+(A+B s) W\left(s+\sigma_{0}\right) \\
= & A K^{-1}+A H^{\prime}\left(s I-F+\sigma_{0} I\right)^{-1} G \\
& +B H^{\prime}\left[\left(s I-F+\sigma_{0} I\right)+F-\sigma_{0} I\right]\left(s I-F+\sigma_{0} I\right)^{-1} G \\
= & \left(A K^{-1}+B H^{\prime} G\right)+\left[A H^{\prime}+B H^{\prime}\left(F-\sigma_{0} I\right)\right]\left(s I-F+\sigma_{0} I\right)^{-1} G
\end{aligned}
$$

and we see that since $Z_{1}(s)$ is positive real, the theorem of (3) may be applied to the triple $\left\{F-\sigma_{0} I, G, H A+\left(F^{\prime}-\sigma_{0} I\right) H B\right\}$, which is a minimal realization of $Z_{1}(s)-Z_{1}(\infty)$. Thus, there exist a positive definite symmetric $P$, and matrices $L$ and $W_{0}$ such that

$$
\begin{aligned}
P\left(F-\sigma_{0} I\right)+\left(F^{\prime}-\sigma_{0} I\right) P & =-L L^{\prime} \\
P G & =H A+\left(F^{\prime}-\sigma_{0} I\right) H B-L W_{0} \\
W_{0}^{\prime} W_{0} & =2 A K^{-1}+B H^{\prime} G+G^{\prime} H B
\end{aligned}
$$

Consider as a tentative Lyapunov function for the system of Fig. 1:

$$
V(x)=x^{\prime} P x+2 \int_{0}^{y} \mu^{\prime}(\rho) B d \rho
$$

where $x$ is the state vector of the system. Observe that the positive definiteness of $P$, the positive semidefiniteness of $B$, and the restrictions on $\mu$ of (2) ensure that $V$ is positive for all nonzero $x$. Differentiating (11) gives

$$
\dot{V}(x)=\dot{x}^{\prime} P x+x^{\prime} P \dot{x}+2 \mu^{\prime}(y) \dot{y} .
$$


The time derivatives may be expressed in terms of $F, G, H^{\prime}$ and $\mu(y)$ by noting that the system of Fig. 1 may be represented as a linear system using state space notation, i.e., from (9)

Thus,

$$
\begin{aligned}
& \dot{x}=F x-G \mu(y) \\
& y=H^{\prime} x .
\end{aligned}
$$

$$
\dot{V}(x)=\left[x^{\prime} F^{\prime}-\mu^{\prime}(y) G^{\prime}\right] P x+x^{\prime} P[F x-G \mu(y)]+2 \mu^{\prime}(y) B H^{\prime}[F x-G \mu(y)] .
$$

Applying (10) and arranging terms, gives

$$
\begin{array}{r}
\dot{V}(x)=-\left[x^{\prime} L-\mu^{\prime}(y) W_{0}^{\prime}\right]\left[L^{\prime} x-W_{0 \mu}(y)\right]-2 \mu^{\prime}(y) A\left[y-K^{-1} \mu(y)\right] \\
+2 \sigma_{0}\left[x^{\prime} P x+\mu^{\prime} B y\right] .
\end{array}
$$

The first term is plainly nonpositive, and the nonnegative nature of $A$ together with the nonlinearity condition (2) ensure the nonpositivity of the second term.

Consequently for the case $\sigma_{0}=0, V(x)$ is, in fact, a Lyapunov function and the first part of the theorem is proved. In order to prove the second part, consider the ratio of $\dot{V}(x)$ to $V(x)$ :

$$
\dot{V}(x) / V(x)=\dot{V}(x) /\left.V(x)\right|_{\sigma_{0}=0}+2 \sigma_{0}\left[x^{\prime} P x+\mu^{\prime} B y\right] /\left(x^{\prime} P x+2 \int_{0}^{\nu} \mu^{\prime} B d \rho\right) .
$$

Since $\dot{V}(x) /\left.V(x)\right|_{\sigma_{0}=0} \leq 0$, for the more general case (16) yields

$$
\dot{V}(x) / V(x) \leq 2 \sigma_{0}\left[x^{\prime} P x+\mu^{\prime} B y\right] /\left(x^{\prime} P x+2 \int_{0}^{y} \mu^{\prime} B d \rho\right) .
$$

Applying condition (5) gives

$$
\dot{V}(x) / V(x) \leq \sigma_{0}
$$

and applying condition (7) gives

$$
\dot{V}(x) / V(x) \leq 2 \sigma_{0}
$$

thus proving the second part of the theorem.

\section{Concluding Remarks}

For the single variable case, Part (a) of the theorem reduces to the original Popov criterion (5) and Part (b) reduces essentially to the result of Yakubovich (4). 
For the special case when the matrix $A$ is taken as the identity matrix 1 , the criterion reduces to that given in (1), and when $A$ is $\alpha I$ and $B$ is $\beta I$ ( $\alpha$ and $\beta$ being real positive numbers), the criterion reduces to that used in (2). Note that if, as in (2), the nonlinearities are permitted to be coupled, the integral in (11) is, in general, dependent on the path of integration (and thus is not well defined); hence, the stability criterion cannot be valid for this more general case using the above theory.

For the special case when $W\left(s+\sigma_{0}\right)$ is itself positive real, $B$ may be chosen as a zero matrix and $A$ the identity matrix, and it may be concluded that the system is stable for any memoryless nonlinearities bounded within the first and third quadrants. An application of this particular case is used to consider the effects of nonlinearities in the transducers of linear optimal control systems, (7).

As remarked in (2), the asymptotic stability of the closed loop system, as distinct merely from its stability, depends on $\dot{V}$. Various conditions ensure asymptotic stability, for example, nonsingularity of $A$ and inequality signs in (2), or any other condition that forces $\dot{V}$ to be not identically zero along a trajectory.

\section{Acknowledgment}

The authors wish to acknowledge helpful discussions with numerous colleagues, particularly R. Kalman, R. Brockett, D. Siljak, and P. Faurre; the latter first pointed out the ill-definition of the integral (8) in the coupled nonlinearity case, and the necessity to not introduce a zero in $Z(s)$ which might cancel a pole of $W(s)$.

\section{References}

(1) H. Tokumaru and N. Saito, "On the Absolute Stability of Automatic Control Systems with Many Nonlinear Characteristics," Memoirs of the Faculty of Engineering, Kyoto Univ., Japan, Vol. XXVII, Part 3, pp. 347-379, July, 1965.

(2) B. D. O. Anderson, "Stability of Control Systems with Multiple Nonlinearities," $J$. Franklin Inst., Vol. 281, No. 9 pp. 155-160, Sept., 1966.

(3) B. D. O. Anderson, "A System Theory Criterion for Positive Real Matrices," S. I. A. M. Journal, Vol. 5, No. 2, pp. 171-182, 1967.

(4) V. A. Yakubovich, "A Matrix Inequality Method in the Theory of Stability in Nonlinear Control Systems, I, Absolute Stability of Forced Vibrations," Auto. and Remote Control, Vol. 25, No. 7, pp. 905-917, 1964.

(5) V. M. Popov, "Absolute Stability of Nonlinear Systems of Automatic Control," Auto. and Remote Control, Vol. 22, No. 8, pp. 857-875, Mar. 1962.

(6) R. W. Newcomb, "Linear Multiport Synthesis," New York, McGraw-Hill Book Co., 1966.

(7) J. B. Moore and B. D. O. Anderson, "Applications of the Multivariable Popov Criterion," Intern. J. of Control, Vol. 5, No. 4, pp. 345-353, 1967. 